\title{
SCALING PROPERTIES OF INFINITELY FLAT CURVES AND SURFACES
}

\section{Alex Iosevich}

\begin{abstract}
We shall give simple sufficient conditions for the Orlicz type bounds for the averaging operators and restriction operators associated with infinitely flat curves in the plane. Our results, obtained by scaling, can be used to recover, up to the endpoints, the results previously obtained in [BMO91], [Bak94], and [Bak94-2]. We also prove some three dimensional analogs of those results.
\end{abstract}

\section{INTRODUCTION}

Let $\gamma \in C^{\infty}([0, \infty)), \gamma(0)=\gamma^{\prime}(0)=0, \gamma^{\prime \prime}(s) \geq 0$, and $\gamma^{\prime \prime}(s)=0$ iff $s=0$. Let

$$
T_{\gamma} f(x)=\int_{0}^{2} f\left(x_{1}-s, x_{2}-\gamma(s)\right) d s=f * \mu(x) .
$$

If $\gamma^{\prime \prime}(s)>0$ on $[0,2]$, it is well known that $T_{\gamma}: L^{p}\left(\mathbb{R}^{2}\right) \rightarrow L^{q}\left(\mathbb{R}^{2}\right)$ if and only if $\left(\frac{1}{p}, \frac{1}{q}\right)$ is contained in the triangle with endpoints $(0,0),(1,1)$ and $\left(\frac{2}{3}, \frac{1}{3}\right)$. (See [Str70], [Litt73]). If $\gamma^{\prime \prime}$ vanishes of order $m-2, m \geq 2$, then $T_{\gamma}: L^{p}\left(\mathbb{R}^{2}\right) \rightarrow L^{q}\left(\mathbb{R}^{2}\right)$ if and only if $\left(\frac{1}{p}, \frac{1}{q}\right)$ is contained in the trapezoid with the endpoints $(0,0),(1,1),\left(\frac{2}{m+1}, \frac{1}{m+1}\right)$, and $\left(\frac{m}{m+1}, \frac{m-1}{m+1}\right)$. (See [RiSt88]).

If $\gamma^{\prime \prime}$ vanishes of infinite order, the estimate $T_{\gamma}: L^{p}\left(\mathbb{R}^{2}\right) \rightarrow L^{q}\left(\mathbb{R}^{2}\right)$ may not hold for any $q>p$. However, the Orlicz space estimates may be possible. For example, in [BMO91], the authors showed that for some flat curves in $\mathbb{R}^{2}$ there exists a Young's function $\Phi$, with $\lim _{t \rightarrow \infty} \frac{\Phi(t)}{t^{2}}=0$ such that the estimate

$$
\left\|T_{\gamma} f\right\|_{L^{2}\left(\mathbb{R}^{2}\right)} \leq C\|f\|_{L^{\Phi}\left(\mathbb{R}^{2}\right)}
$$

holds, where $L^{\Phi}\left(\mathbb{R}^{2}\right)$ denotes the standard Orlicz space, associated to an increasing Young function $\Phi$, equipped with the norm

$$
\|f\|_{\Phi} \equiv \inf \left\{s>0: \int \Phi\left(\frac{|f(x)|}{s}\right) d x \leq 1\right\} .
$$

More precisely, the result proved in [BMO91] is the following.

Research at MSRI supported in part by NSF grant DMS97-06825 
Theorem I. (Bak, McMichael, and Oberlin [BMO91]) Let $T_{\gamma}$ be as above. If there exist constants $d \in(0,2], \epsilon>0$, and $\beta>0$ such that

$$
\int_{0}^{\epsilon} D(\beta s)(\gamma(s))^{\frac{2}{d}} s^{3(2 / d-1)} d s<\infty,
$$

then there exists a constant $C$ and a Young function $\Phi$ with $\Phi(t) \approx\left(t H_{+}^{-1}\left(t^{-d}\right)\right)^{2}$ such that $T_{\gamma}: L^{\Phi}\left(\mathbb{R}^{2}\right) \rightarrow L^{2}\left(\mathbb{R}^{2}\right)$, where $H_{+}^{-1}(x)=1$ for $x>1, H^{-1}(x)$ for $x \leq 1$ with $H(x)=x^{3} \gamma(x)$, and $D(s)=\left|\left\{\xi \in \mathbb{R}^{2}:|\widehat{\mu}(\xi)|>s\right\}\right|$.

In [Bak94] the following three dimensional result was proved.

Theorem II. (Bak [Bak94]) Let

$$
T_{\gamma}^{n} f(x)=\int_{\{0 \leq|y| \leq 2\}} f\left(x^{\prime}-y, x_{n}-\gamma(|y|)\right) d y,
$$

where $\left(x^{\prime}, x_{n}\right) \in \mathbb{R}^{n-1} \times \mathbb{R}, \gamma(0)=\gamma^{\prime}(0)=\gamma^{\prime \prime}(0)=0, \gamma^{\prime \prime}(s)>0$ for $s>0$, and $\frac{\gamma^{\prime}(s)}{s}$ is non-decreasing for $s>0$. Let $\gamma_{n}(s)=t^{n} \gamma^{\prime}(s)$. Let $n+1 \leq q<\infty$ and assume that for some $\delta>0$ and $c=c(q)>0 \frac{\gamma_{n}(s)}{s^{q+\delta}}$ is non-decreasing for $0<s \leq c$. Let $\Phi=\Phi_{q}$ be a Young's function such that $\Phi^{-1}(s) \approx \Psi^{-1}(s)=\frac{s^{\frac{1}{q}}}{\left(\gamma_{n}^{-1}(1 / s)\right)^{n-1}}$ if $s \geq 1$, and $\Psi^{-1}(s)=s^{\frac{1}{q}}$ if $s<1$. If $n=2$ or 3 there exists a constant $C$ such that for every Borel set $E \subset \mathbb{R}^{n}$ with $|E|<\infty$ $T_{\gamma}^{n}: L^{\Phi}\left(\mathbb{R}^{n}\right) \rightarrow L^{q}\left(\mathbb{R}^{n}\right), f=\chi_{E}$, the characteristic function of $E$.

In this paper we shall give a simple set of sufficient, and in many cases, necessary conditions, such that $T_{\gamma}: L^{\Phi}\left(\mathbb{R}^{2}\right) \rightarrow L^{\Psi}\left(\mathbb{R}^{2}\right)$. We shall also see that the techniques of this paper can be used to obtain $L^{\Phi}\left(\mathbb{R}^{2}\right) \rightarrow L^{2}\left(\mathbb{R}^{2}\right)$ bounds for the restriction operator

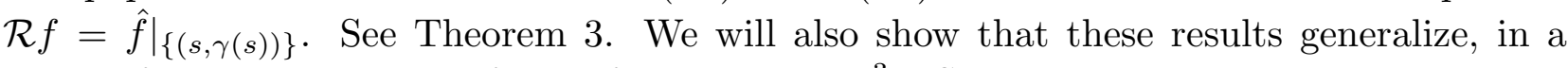
straightforward way, to surfaces of rotation in $\mathbb{R}^{3}$. See the section "Three dimensions" below. Our result for the restriction operator is motivated by the following result due to Bak.

Theorem III. (Bak, [Bak94-2]) Suppose that $\frac{\gamma(s)}{s^{3}}$ is increasing on $[0,2]$. Suppose that $\frac{\gamma^{\prime \prime}(s)}{s}$ is increasing on $(0, \delta)$ for some $\delta>0$. Then for $1 \leq q<\infty$ and $0<d<1$ there exists a constant $C=C_{d, q}$ such that for all $f \in \mathcal{S}\left(\mathbb{R}^{2}\right)$, the class of rapidly decreasing functions,

$$
\left(\int_{0}^{\delta}|\hat{f}(s, \gamma(s))|^{q} d s\right)^{\frac{1}{q}} \leq C+C \int_{\mathbb{R}^{2}}|f(x)| \cdot\left[\gamma_{q}^{-1}(|f(x)|)\right]^{d} d x,
$$

where $\gamma_{q}(s)=s^{q-1} \gamma\left(s^{q}\right)$.

The main idea behind our two-dimensional results is the following lemma motivated by the results in [CCVWW89] where it was observed that even though finite type convex curves (e.g. $\left\{\left(s, s^{m}\right):-1 \leq s \leq 1\right\}$ ) behave very well under the usual diagonal scaling of the form $\left(x_{1}, x_{2}\right) \rightarrow\left(2^{j} x_{1}, 2^{m j} x_{2}\right)$, infinite type curves (e.g. $\left.\left\{\left(s, e^{-1 / s^{2}}\right):-1 \leq s \leq 1\right\}\right)$ require a more careful non-diagonal scaling. 
Lemma 1. Let $\tau_{j} f(x)=f\left(2^{-j} x_{1}, \gamma_{j} x_{1}+h_{j} x_{2}\right)$, where $\gamma_{j}=\gamma\left(2^{-j}\right)$ and $h_{j}$ is defined as above. Let

$$
T_{j}^{*} f(x)=2^{j} \tau_{j} T_{j} \tau_{j}^{-1} f(x)
$$

where

$$
T_{j} f(x)=\int_{2^{-j}}^{2^{-j+1}} f\left(x_{1}-s, x_{2}-\gamma(s)\right) d s
$$

Then $T_{j}^{*}: L^{p}\left(\mathbb{R}^{2}\right) \rightarrow L^{q}\left(\mathbb{R}^{2}\right)$ for $\left(\frac{1}{p}, \frac{1}{q}\right) \in \mathcal{T}$ with constants independent of $j$.

Proof. Since $T_{j}^{*}$ clearly maps $L^{1} \rightarrow L^{1}$ and $L^{\infty} \rightarrow L^{\infty}$, with constants independent of $j$, it suffices to check that $T_{j}^{*}: L^{\frac{3}{2}}\left(\mathbb{R}^{2}\right) \rightarrow L^{3}\left(\mathbb{R}^{2}\right)$ with constants independent of $j$. The classical proof of the $L^{p}$ improving properties of measures supported on surfaces with non-vanishing Gaussian curvature (see e.g. [Str70] and the subsection "Simplification" below) shows that it suffices to check that if we write $T_{j}^{*} f(x)=f * \nu_{j}(x)$, then

$$
\left|\hat{\nu_{j}}(\xi)\right| \leq C(1+|\xi|)^{-\frac{1}{2}}
$$

with $C$ independent of $j$. Then

Let $T_{j} f(x)=f * \mu_{j}(x)$. Let $A_{j}$ denote the matrix given by the equation $\tau_{j} f(x)=f\left(A_{j} x\right)$.

$$
f * \nu_{j}(x)=2^{j} \mu_{j} * \tau_{j}^{-1} f\left(A_{j} x\right) .
$$

Using the elementary properties of the Fourier transform,

$$
\hat{f}(\xi) \hat{\nu_{j}}(\xi)=2^{j} \hat{f}\left(\left(A_{j}^{-1}\right)^{t} A_{j}^{t} \xi\right) \hat{\mu_{j}}\left(\left(A_{j}^{-1}\right)^{t} \xi\right)=2^{j} \hat{f}(\xi) \hat{\mu}_{j}\left(\left(A_{j}^{-1}\right)^{t} \xi\right),
$$

since $\left(A_{j}^{-1}\right)^{t} A_{j}^{t}=I$, where $A_{j}^{t}$ denotes the transpose matrix of $A_{j}$.

By Proposition 4.2 of [CCVWW89],

$$
2^{j}\left|\hat{\mu}_{j}\left(\left(A_{j}^{-1}\right)^{t} \xi\right)\right| \leq C\left(1+\left|\left(A_{j}^{-1}\right)^{t} A_{j}^{t} \xi\right|\right)^{-\frac{1}{2}}=C(1+|\xi|)^{-\frac{1}{2}} .
$$

Diving both sides of (11) by $\hat{f}(\xi)$ and using (12) completes the proof of Lemma 1.

The main idea behind the three dimensional results in this paper is the estimate of the Fourier transform of the measure carried by a family of radial surfaces dependent on a parameter. See Lemma 6 below. It turns out that while it is difficult to find a scaling that gives the optimal decay in all directions, even the most naive scaling allows one to get the 
optimal decay in the direction normal to the surface at the origin. This turns out to be enough to obtain the desired results for the averaging and restriction operators.

Our plan is as follows. In the section titled "Scaling" we shall give sufficient conditions for the $L^{\Phi}\left(\mathbb{R}^{2}\right) \rightarrow L^{\Psi}\left(\mathbb{R}^{2}\right)$ boundedness of the operator $T_{\gamma}$ and the $L^{\Phi}\left(\mathbb{R}^{2}\right) \rightarrow L^{2}\left(\mathbb{R}^{2}\right)$ boundedness of the restriction operator $\mathcal{R}$ in terms of the Orlicz norms of the family of dilation operators of the form $\tau_{A} f(x)=f(A x)$ where $A$ is an invertible matrix.

In the subsection titled "Simplification" we will show that the assumptions of our main result can be simplified if we are willing to assume that $\sup _{0<a<b} \frac{\gamma^{\prime \prime}(a)}{\gamma^{\prime \prime}(b)} \leq C$.

In the subsection titled "Three dimensions" we shall extend our results to radial surfaces in $\mathbb{R}^{3}$.

In the section titled "Orlicz norms of dilation operators" we shall compute upper and lower bounds for the Orlicz norms of the operators $\tau_{A}$ under various assumptions on the Young functions in question.

We shall conclude the paper with the subsection on examples.

\section{SCALING}

The following definition is motivated by interpolation results for $L^{p}$ and Orlicz spaces (see e.g. [Tor76], [Tor86]).

Definition 2. Let $\mathcal{T}$ denote the triangle with the endpoints $(0,0),(1,1)$, and $\left(\frac{2}{3}, \frac{1}{3}\right)$. We say that $(\Phi, \Psi) \subset \mathcal{T}$ if every linear operator bounded from $L^{p}\left(\mathbb{R}^{2}\right) \rightarrow L^{q}\left(\mathbb{R}^{2}\right),\left(\frac{1}{p}, \frac{1}{q}\right) \in \mathcal{T}$, is bounded from $L^{\Phi}\left(\mathbb{R}^{2}\right) \rightarrow L^{\Psi}\left(\mathbb{R}^{2}\right)$.

Let $\mathcal{T}^{\prime}=\left\{p: 1 \leq p \leq \frac{6}{5}\right\}$. We say that $\Phi \subset \mathcal{T}^{\prime}$ if every linear operator bounded from $L^{p}\left(\mathbb{R}^{2}\right) \rightarrow L^{2}\left(\mathbb{R}^{2}\right), p \in \mathcal{T}^{\prime}$, is bounded from $L^{\Phi}\left(\mathbb{R}^{2}\right) \rightarrow L^{2}\left(\mathbb{R}^{2}\right)$.

Our main results are the following.

Theorem 3. Let $T_{\gamma}$ be as above. Suppose that there exists $\epsilon>0$ so that $h^{\prime}(t)>\epsilon \frac{h(t)}{t}$ for every $t>0$, where $h(t)=t \gamma^{\prime}(t)-\gamma(t)$. Let $(\Phi, \Psi) \subset \mathcal{T}$.

Let $\tau_{j} f(x)=f\left(2^{-j} x_{1}, \gamma_{j} x_{1}+h_{j} x_{2}\right)$ where $\gamma_{j}=\gamma\left(2^{-j}\right)$ and $h_{j}=h\left(2^{-j}\right)$. Let $N_{j}(\Phi)$ denote the $\left(L^{\Phi}\left(\mathbb{R}^{2}\right), L^{\Phi}\left(\mathbb{R}^{2}\right)\right)$ norm of the operator $\tau_{j}$, and let $N_{j}^{-1}(\Psi)$ denote the $\left(L^{\Psi}\left(\mathbb{R}^{2}\right), L^{\Psi}\left(\mathbb{R}^{2}\right)\right)$ norm of the operator $\tau_{j}^{-1}$.

Suppose that

$$
\sum_{j=0}^{\infty} 2^{-j} N_{j}(\Phi) N_{j}^{-1}(\Psi)<\infty .
$$

Then $T_{\gamma}: L^{\Phi}\left(\mathbb{R}^{2}\right) \rightarrow L^{\Psi}\left(\mathbb{R}^{2}\right)$.

Remark. Note that by duality it suffices to check $T_{\gamma}: L^{\Psi^{*}}\left(\mathbb{R}^{2}\right) \rightarrow L^{\Phi^{*}}\left(\mathbb{R}^{2}\right)$. In other words, it suffices to check the condition (13) with $N_{j}(\Phi)$ replaced by $N_{j}\left(\Psi^{*}\right)$, and $N_{j}^{-1}(\Psi)$ replaced by $N_{j}^{-1}\left(\Phi^{*}\right)$. 
Theorem 4. Let $\mathcal{R} f$ be defined as above. Let $\gamma$ satisfy the assumptions of Theorem 3. Let $\sigma_{j} f(x)=\left|A_{j}\right| f\left(A_{j}^{t} x\right)$ with $A_{j}$ defined by $\tau_{j} f(x)=f\left(A_{j} x\right)$, where $\tau_{j}$ is as above. Let $\mathcal{N}_{j}^{-1}(\Phi)$ denote the $\left(L^{\Phi}\left(\mathbb{R}^{2}\right), L^{\Phi}\left(\mathbb{R}^{2}\right)\right)$ norm of the operator $\sigma_{j}^{-1}$. Let $\Phi \in \mathcal{T}^{\prime}$.

Suppose that

$$
\sum_{j=0}^{\infty} 2^{-\frac{j}{2}} \mathcal{N}_{j}^{-1}(\Phi)<\infty .
$$

Then $\mathcal{R}: L^{\Phi}\left(\mathbb{R}^{2}\right) \rightarrow L^{2}(\Gamma)$, where $\Gamma=\{(s, \gamma(s)): 0 \leq s \leq 2\}$.

We shall now prove Theorem 3 and Theorem 4 .

\section{Proof of Theorem 3}

Let $T_{j}, T_{j}^{*}$ be defined as above. Then

$$
\left\|T_{j} f\right\|_{\Psi}=2^{-j}\left\|\tau_{j}^{-1} T_{j}^{*} \tau_{j} f\right\|_{\Psi}
$$

By Lemma $1, T_{j}^{*}: L^{p}\left(\mathbb{R}^{2}\right) \rightarrow L^{q}\left(\mathbb{R}^{2}\right)$ for $\left(\frac{1}{p}, \frac{1}{q}\right) \in \mathcal{T}$ with constants independent of $j$. By definition of $N_{j}(\Phi)$ and $N_{j}^{-1}(\Psi)$ it follows that $T: L^{\Phi}\left(\mathbb{R}^{2}\right) \rightarrow L^{\Psi}\left(\mathbb{R}^{2}\right)$ if $(13)$ holds. This completes the proof of Theorem 3 .

\section{Proof of Theorem 4}

The proof of Theorem 4 is along the same lines as the proof of Theorem 3. Let $\mathcal{R}_{j} f=\left.\hat{f}\right|_{\Gamma_{j}}$, where $\Gamma_{j}=\left\{(s, \gamma(s)): 2^{-j} \leq s \leq 2^{-j+1}\right\}$. Let $\sigma_{j} f(x)=\left|A_{j}\right| f\left(A_{j}^{t} x\right)$. Using the elementary properties of the Fourier transform it is not hard to see that $\mathcal{R}_{j} \sigma_{j} f=\hat{f}\left(A_{j}^{-1} \Gamma_{j}(s)\right)$. It follows that

$$
\left\|\mathcal{R}_{j} \sigma_{j} f\right\|_{2}=2^{-\frac{j}{2}} \| \hat{f}\left(A_{j}^{-1} \Gamma_{0}\left(2^{-j}\right) \|_{2} .\right.
$$

By Proposition 4.2 in [CCVWW89], the Fourier transform of the Lebesgue measure on $A_{j}^{-1} \Gamma_{0}\left(2^{-j}\right.$.) is bounded by $C(1+|\xi|)^{-\frac{1}{2}}$, where $C$ is independent of $j$. The proof of the restriction theorem for curves with non-vanishing Gaussian curvature (see e.g. [St93]) implies that

$$
\left\|\mathcal{R}_{j} \sigma_{j} f\right\|_{2} \leq C 2^{-\frac{j}{2}}\|f\|_{\Phi},
$$

for $\Phi \subset \mathcal{T}^{\prime}$. It follows that

$$
\left\|\mathcal{R}_{j} f\right\|_{2} \leq C 2^{-\frac{j}{2}}\left\|\sigma_{j}^{-1} f\right\|_{\Phi} .
$$

This completes the proof of Theorem 4 . 
Simplification. It is not hard to see that the statements of Theorem 3 and Theorem 4 can be simplified if we are willing to assume that $\gamma^{\prime \prime}$ is increasing on $[0,2]$, or, even, that $\sup _{0<a \leq b} \frac{\gamma^{\prime \prime}(b)}{\gamma^{\prime \prime}(a)} \geq C$. More precisely, under this assumption, everywhere in the statements of Theorem 3 and Theorem 4, we can replace the scaling transformation $\tau_{j} f(x)=f\left(2^{-j} x_{1}, \gamma_{j} x_{1}+h_{j} x_{2}\right)$ by a simpler scaling transformation $\tau_{j}^{\prime} f(x)=f\left(2^{-j} x_{1}, \gamma_{j} x_{2}\right)$. This is the consequence of the following rephrasing of the classical $L^{p}$-improving result for curves with everywhere non-vanishing curvature and the Stein-Tomas restriction theorem in $\mathbb{R}^{2}$.

Lemma 5. Let $T f(x)=\int_{I} f\left(x_{1}-s, x_{2}-\phi(s)\right) d s, \mathcal{R} f=\left.\hat{f}\right|_{\{(s, \phi(s)): s \in I\}}$, where $I$ is a compact interval and $\phi$ is a smooth function. Suppose that $\phi^{\prime \prime}(s) \geq 1$ on $I$. Then $T: L^{p}\left(\mathbb{R}^{2}\right) \rightarrow$ $L^{q}\left(\mathbb{R}^{2}\right),\left(\frac{1}{p}, \frac{1}{q}\right) \in \mathcal{T}$, and $\mathcal{R}: L^{p}\left(\mathbb{R}^{2}\right) \rightarrow L^{p}\left(\mathbb{R}^{2}\right), p \in \mathcal{T}^{\prime}$, with constants independent of $\phi$.

Before proving the lemma, let's see how it implies the claim in the first paragraph. The analog of the operator $T_{j}^{*}$ in the proof of Theorem 3 is just the averaging operator over the curve $\left(s, \phi_{j}(s)\right), 1 \leq s \leq 2$, where $\phi_{j}(s)=\frac{\gamma\left(2^{-j} s\right)}{\gamma_{j}}$. By assumption and the mean value theorem $\phi_{j}^{\prime \prime}(s) \geq 1$, so Lemma 5 implies that the new $T_{j}^{*}: L^{p}\left(\mathbb{R}^{2}\right) \rightarrow L^{q}\left(\mathbb{R}^{2}\right),\left(\frac{1}{p}, \frac{1}{q}\right) \in \mathcal{T}$. The rest of the proof goes through as before and we get the claim in the first paragraph. The argument in the restriction theorem is basically the same.

We now prove Lemma 5. Again, we prove the case of the $L^{p}$-improving theorem, the restriction proof being similar. Let $K_{z}(x)=\frac{1}{\Gamma(z)}\left(x_{2}-\phi\left(x_{1}\right)\right)^{z-1} \chi_{I}\left(x_{1}\right)$, where $\Gamma$ denotes the standard gamma function and $\chi_{I}$ is the characteristic function of the interval $I$. Let $T_{z} f(x)=f * K_{z}(x)$. It is clear that if $\operatorname{Re}(z)=1$, then $T_{z}: L^{1}\left(\mathbb{R}^{2}\right) \rightarrow L^{\infty}\left(\mathbb{R}^{2}\right)$ with universal constants. It remains to show that when $\operatorname{Re}(z)=-\frac{1}{2}$, then $T_{z}: L^{2}\left(\mathbb{R}^{2}\right) \rightarrow L^{2}\left(\mathbb{R}^{2}\right)$ with constants independent of $\phi$. Let's compute $m_{z}(\xi)=\hat{K}_{z}(\xi)$. Well,

$$
m_{z}(\xi)=\int e^{-i\langle x, \xi\rangle} K_{z}(x) d x
$$

Let $y_{1}=x_{1}, y_{2}=x_{2}-\phi\left(x_{1}\right)$. We get

$$
\frac{1}{\Gamma(z)} \iint_{I} e^{-i\left(y_{1} \xi_{1}+\phi\left(y_{1}\right) \xi_{2}\right)} e^{-i y_{2} \xi_{2}} y_{2}^{z-1} d y_{1} d y_{2} \text {. }
$$

When $\operatorname{Re}(z)=-\frac{1}{2}$, the expression in $(20)$ is controlled by $|\widehat{d \sigma}(\xi)|\left|\xi_{2}\right|^{\frac{1}{2}}$, so, by Plancherel's theorem it remains to show that $|\widehat{d \sigma}(\xi)| \leq C\left|\xi_{2}\right|^{-\frac{1}{2}}$ with constants independent of $\phi$. This immediately follows by the van der Corput lemma since $\phi^{\prime \prime}(s) \geq 1$ on $I$.

Three dimensions. In this section we shall see that if $S=\left\{x=\left(x^{\prime}, x_{3}\right) \in \mathbb{R}^{3}: x_{3}=\right.$ $\left.\gamma\left(\left|x^{\prime}\right|\right)\right\}$, where $\gamma$ satisfies the assumptions of the previous subsection (Simplification), then one can easily generalize Theorem 3 and Theorem 4 to three dimensions by replacing the scaling transformation $\tau_{j}^{\prime}$ by its three dimensional version, $\tau_{j}^{\prime} f(x)=f\left(2^{-j} x^{\prime}, \gamma_{j} x_{3}\right)$, using the following three dimensional version of Lemma 5. 
Lemma 6. Let $T f(x)=\int_{B} f\left(x^{\prime}-y, x_{n}-G(y)\right) d y, \mathcal{R} f(x)=\left.\hat{f}\right|_{\{(y, G(y)): y \in B\}}$, where $B$ is the annulus $\{y: 1 \leq|y| \leq 2\}$, and $G(y)=\phi(|y|)$. Suppose that $\min \left\{\phi^{\prime}, \phi^{\prime \prime}\right\} \geq 1$ on B. Then $T: L^{p}\left(\mathbb{R}^{3}\right) \rightarrow L^{q}\left(\mathbb{R}^{3}\right)$ for $\left(\frac{1}{p}, \frac{1}{q}\right) \in \mathcal{T}_{3}$, the triangle with the endpoints $(0,0),(1,1)$, and $\left(\frac{3}{4}, \frac{1}{4}\right)$, and $\mathcal{R}: L^{p}\left(\mathbb{R}^{3}\right) \rightarrow L^{2}\left(\mathbb{R}^{3}\right), p \in \mathcal{T}_{3}^{\prime}=\left\{p: 1 \leq p \leq \frac{4}{3}\right\}$, with constants independent of $G$.

As we noted above, we can now prove the obvious analogs of Theorem 3 and Theorem 4. We let $\tau_{j} f(x)=f\left(2^{-j} x^{\prime}, \gamma_{j} x_{3}\right)$. The analog of the operator $T_{j}^{*}$ is the averaging operator over the hypersurface $\left\{x: 1 \leq\left|x^{\prime}\right| \leq 2 ; \quad x_{3}=G_{j}\left(x^{\prime}\right)\right\}$, where $G_{j}\left(x^{\prime}\right)=\frac{\gamma\left(2^{-j}\left|x^{\prime}\right|\right)}{\gamma_{j}}$. The determinant of the hessian matrix of $G_{j}$ is $2^{-2 j} \gamma^{\prime \prime}\left(2^{-j} r\right)\left(\frac{2^{-j} \gamma^{\prime}\left(2^{-j} r\right)}{r}\right)$, where $r=\left|x^{\prime}\right|$. By assumption and the mean value theorem this quantity is bounded below by 1 . The rest of the argument is the same as the proof of Theorem 3, with Lemma 6 replacing Lemma 1. The argument for the restriction operator is similar.

We shall now prove Lemma 6 . By the proof of Lemma 5 it suffices to show that

$$
|F(\xi)|=\left|\int_{B} e^{i\left(\left\langle x^{\prime}, \xi\right\rangle+\xi_{3} G\left(x^{\prime}\right)\right)} d x^{\prime}\right| \leq C\left|\xi_{3}\right|^{-1}
$$

with $C$ independent of $G$.

Going into polar coordinates, applying standard stationary phase, and making a change of variables sending $r \rightarrow r \xi_{3}^{-\frac{1}{2}}$, we get $\xi_{3}^{-1}$ times

$$
I(A, t)=\int_{t^{\frac{1}{2}}[1,2]} e^{i\left(r A t^{-\frac{1}{2}}-t \phi\left(t^{-\frac{1}{2}} r\right)\right)} r^{1} b\left(r A t^{-\frac{1}{2}}\right) d r,
$$

where $A=\left|x^{\prime}\right|, t=\xi_{3}$, and $b$ is a symbol of order $-\frac{1}{2}$. It suffices to prove that $I$ is uniformly bounded with constants independent of $A, t$, and $\phi$.

Suppose that either $A \approx|t|$ or $A>>|t|$. Now, by the van der Corput lemma, the integral $I(A, t)$ is bounded by

$$
C\left|t^{\frac{1}{2}} b(A)\right|
$$

where $C$ is a universal constant, since the second derivative of the phase function of $I$, $\phi^{\prime \prime}\left(r t^{-\frac{1}{2}}\right)$, is bounded below by 1 on $\left[t^{\frac{1}{2}}, 2 t^{\frac{1}{2}}\right]$ by assumption. The expression in (23) is bounded above by another universal constant $C^{\prime}$ since $b$ is a symbol of order $-\frac{1}{2}$, and $|t| \leq A$.

It remains to handle the case when $A<<t$. We undo the change of variables sending $r \rightarrow r t^{-\frac{1}{2}}$, and we let $h(r)=r A-t \phi(r)$. It is not hard to see that $\left|h^{\prime}(t)\right| \geq|A-t| \geq|t|$. Van der Corput lemma gives us the decay $\frac{C}{t}$ and the proof of Lemma 6 is complete. 


\section{ORLICZ NORMS OF DILATION OPERATORS}

Lemma 7. Let $\Phi$ be a Young function such that

$$
\Phi(a) \Phi(b) \leq \Phi(C a b)
$$

for all $a, b>0$ with some $C>0$. Let $\operatorname{det}(A)=t$. Then

$$
\frac{c}{\Phi^{-1}(t)}\|f\|_{\Phi} \leq\left\|\tau_{A} f\right\|_{\Phi} \leq C \Phi^{-1}\left(\frac{1}{t}\right)\|f\|_{\Phi}
$$

Lemma 8. Let $\Phi$ be a Young function such that

$$
\Phi(a) \Phi(b) \geq \Phi(C a b)
$$

for all $a, b>0$ with some $C>0$. Then

$$
c \Phi^{-1}\left(\frac{1}{t}\right)\|f\|_{\Phi} \leq\left\|\tau_{A}\right\|_{\Phi} \leq \frac{C}{\Phi^{-1}(t)}\|f\|_{\Phi} .
$$

Lemma 9. Let $\Phi^{*}$ denote the conjugate function of $\Phi$ given by the equation

$$
\Phi^{*}(s)=\inf _{t}(t s-\Phi(t)) .
$$

Let $N_{A}(\Phi)$ denote the $\left(L^{\Phi}, L^{\Phi}\right)$ norm of the operator $\tau_{A}$. Then

$$
\frac{1}{t} \leq N_{A}(\Phi) N_{A}\left(\Phi^{*}\right)
$$

\section{Proof of Lemma 7 And Lemma 8}

We must estimate $\inf \{s>0\}$ such that

$$
\int \Phi\left(\frac{|f(A x)|}{s}\right) d x \leq 1,
$$

where we may assume that $\int \Phi(|f(x)|) d x=1$.

Making a change of variables and using (24) this immediately reduces to $\frac{1}{\Phi(s)} \leq t$ which implies that $\Phi(s) \geq \frac{1}{t}$. It follows that $s \geq \Phi^{-1}\left(\frac{1}{t}\right)$. Taking the inf proves the second inequality of (25). Replacing $A$ by $A^{-1}$ we see that the second inequality implies the first. This completes the proof of Lemma 7.

Making a change of variables and using (26) reduces (30) to $\Phi\left(\frac{1}{s}\right) \leq t$ which implies that $s \geq \frac{1}{\Phi^{-1}(t)}$. This proves the second inequality in (27). Replacing $A$ by $A^{-1}$ we see again that the second inequality implies the first. This completes the proof of Lemma 8. 


\section{Proof of Lemma 9}

By Holder's inequality

$$
\left\|\tau_{A} f\right\|_{2}^{2} \leq\left\|\tau_{A} f\right\|_{\Phi}\left\|\tau_{A} f\right\|_{\Phi^{*}}
$$

Since $\left\|\tau_{A} f\right\|_{2}^{2}=\frac{1}{t}\|f\|_{2}$, the conclusion of Lemma 9 follows.

The following is a sample result obtained by combining Theorem 3, Lemma 7 and Lemma 8.

Theorem 10. Let $T_{\gamma}$ be as above. Suppose that $\gamma$ satisfies the conditions of Theorem 3. Suppose that $\Phi$ and $\Psi$ satisfy $(24)$. Then $T_{\gamma}: L^{\Phi}\left(\mathbb{R}^{2}\right) \rightarrow L^{\Psi}\left(\mathbb{R}^{2}\right)$ if

$$
\sum_{j=0}^{\infty} 2^{-j} \Phi^{-1}\left(\frac{2^{j}}{h_{j}}\right) \Psi^{-1}\left(2^{-j} h_{j}\right)<\infty
$$

Suppose that $\Phi$ and $\Psi$ satisfy (26). Then $T_{\gamma}: L^{\Phi}\left(\mathbb{R}^{2}\right) \rightarrow L^{\Psi}\left(\mathbb{R}^{2}\right)$ if

$$
\sum_{j=0}^{\infty} \frac{2^{-j}}{\Phi^{-1}\left(2^{-j} h_{j}\right) \Psi^{-1}\left(\frac{2^{j}}{h_{j}}\right)}<\infty
$$

Suppose that $\Phi$ satisfies (24) and $\Psi$ satisfies $(26)$. Then $T_{\gamma}: L^{\Phi}\left(\mathbb{R}^{2}\right) \rightarrow L^{\Psi}\left(\mathbb{R}^{2}\right)$ if

$$
\sum_{j=0}^{\infty} 2^{-j} \frac{\Phi^{-1}\left(\frac{2^{j}}{h_{j}}\right)}{\Psi^{-1}\left(\frac{2^{j}}{h_{j}}\right)}<\infty
$$

Suppose that $\Phi$ satisfies (26) and $\Psi$ satisfies $(24)$. Then $T_{\gamma}: L^{\Phi}\left(\mathbb{R}^{2}\right) \rightarrow L^{\Psi}\left(\mathbb{R}^{2}\right)$ if

$$
\sum_{j=0}^{\infty} 2^{-j} \frac{\Psi^{-1}\left(2^{-j} h_{j}\right)}{\Phi^{-1}\left(2^{-j} h_{j}\right)}<\infty
$$

Remark. A three dimensional version of this result can be generated using Lemma 6 and the proof of Theorem 3 under the assumption that $\gamma^{\prime \prime}$ is increasing on $[0,2]$ by replacing $h_{j}$ by $\gamma_{j}$ and $2^{j}$ by $2^{2 j}$ in Theorem 10 above.

The following is a sample theorem obtained by combining Theorem 4, Lemma 7 and Lemma 8. 
Theorem 11. Let $\mathcal{R}$ be as above. Suppose that $\gamma$ satisfies the conditions of Theorem 4. Suppose that $\Phi$ satisfies (24). Then $\mathcal{R}: L^{\Phi}\left(\mathbb{R}^{2}\right) \rightarrow L^{2}(\Gamma)$ if

$$
\sum_{j=0}^{\infty} \frac{2^{\frac{j}{2}} \Phi^{-1}\left(2^{-j} h_{j}\right)}{h_{j}}<\infty .
$$

Suppose that $\Phi$ satisfies (26). Then $\mathcal{R}: L^{\Phi}\left(\mathbb{R}^{2}\right) \rightarrow L^{2}(\Gamma)$ if

$$
\sum_{j=0}^{\infty} \frac{2^{\frac{j}{2}}}{h_{j} \Phi^{-1}\left(\frac{2^{j}}{h_{j}}\right)}<\infty .
$$

Remark. Three dimensional analogs can be generated using Lemma 6 and the proof of Theorem 4 by replacing $h_{j}$ by $\gamma_{j}$ and $2^{j}$ by $2^{2 j}$.

\section{EXAMPLES}

Example 1. Let $\Phi(s)=\int_{0}^{s} \phi(t) d t$, where $\phi(t)=t^{q-1} \log ^{-l}(t), l>0$. Let $\Psi(s)=s^{q}$. Let $\gamma(s)=e^{-\frac{1}{s^{\alpha}}}$. A calculation shows that $\Phi(s) \approx s^{q} \log ^{-l}(s)$ and that $\Phi$ satisfies the condition (24). Since $\Psi$ satisfies any condition you want, Theorem 10, along with the subsection "Simplification" implies that $T_{\gamma}: L^{\Phi}\left(\mathbb{R}^{2}\right) \rightarrow L^{\Psi}\left(\mathbb{R}^{2}\right)$ if

$$
\sum_{j=0}^{\infty} 2^{-j} 2^{j \frac{\alpha l}{q}}<\infty,
$$

since $\Phi^{-1}(s) \leq s^{\frac{1}{q}} \log ^{\frac{l}{q}}(s)$ (see [Bak95], Example 1.3).

The sum in (38) converges if $\alpha l<q$.

Example 2. Let $\Phi^{-1}(s) \approx s \log ^{-l}(s)$. Let $\gamma$ be as in Example 1. It is not hard to see that $\Phi$ satisfies (26). Theorem 11 and the subsection "Simplification" imply that $\mathcal{R}: L^{\Phi}\left(\mathbb{R}^{2}\right) \rightarrow$ $L^{2}(\Gamma)$ if

$$
\sum_{j=0}^{\infty} 2^{-\frac{j}{2}} 2^{j \alpha l}<\infty
$$

which takes place if $\alpha l<\frac{1}{2}$.

The necessity results in [Bak94], [Bak94-2], and [BMO91], associated with Theorems I, II, and III in the introduction, show that Examples 1 and 2 give optimal results, at least up to the endpoints. Indeed, to test the sharpness of Example 1, we just test $T_{\gamma}$ against a characteristic of a cube with side-lengths $\delta$ and $\gamma(\delta)$, (see [BMO91]), whereas the sharpness of Example 2 follows by a variant of the classical Knapp homogeneity argument. See [Bak94].

Acknowledgements: The author wishes to thank J. Vance for many helpful conversations and for pointing out the reference [CCVWW89]. 


\section{REFERENCES}

[Bak94] J.-G. Bak, Restrictions of Fourier tranforms to flat curves in $\mathbb{R}^{2}$, Illinois J. Math. 38 (1994).

[Bak94-2] J.-G. Bak, Sharp convolution estimates for measures on flat surfaces, (preprint) (1994).

[Bak95] J.-G. Bak, Averages over surfaces with infinitely flat points, J. Func. Anal. 129 (1995).

[BMO91] J.-G. Bak, D. McMichael, and D. Oberlin, Convolution estimates for some measures on flat curves, J. Func. Anal. 101 (1991).

[CCVWW89] A. Carbery, M. Christ, J. Vance, S. Wainger, and D. Watson, Operators associated to flat plane curves: $L^{p}$ estimates via dilation methods, Duke Math. J. 59 (1989).

[CarSj72] L. Carleson and P. Sjolin, Oscillatory integrals and the multiplier problem for the disc, Studia Math. 44 (1972).

[Litt73] W. Littman, $\left(L^{p}, L^{q}\right)$ estimates for singular integral operators, Proc. Symp. Pure Math. 23 (1973).

[RiSt88] F. Ricci and E. Stein, Harmonic analysis on nilpotent groups and singular integrals II, J. Func. Anal. 78 (1988).

[St93] E. Stein, Harmonic Analysis, Princeton Univ. Press (1993).

[Str70] R. Strichartz, Convolution with kernels having singularities on a sphere, Trans. Amer. Math. Soc. 148 (1970).

[Tom75] P. Tomas, A restriction theorem for the Fourier transform, Bull. Amer. Math. Soc. 81 (1975).

[Tor76] A. Torchinsky, Interpolation of operators and Orlicz classes, Studia Math. 59 (1976).

[Tor86] A. Torchinsky, Real variable methods in harmonic analysis, Academic Press, Orlando (1986).

Department of Mathematics Wright State University Dayton Ohio; email: iosevich @ZARA.MATH.WRIGHT.EDU

Current address: Mathematical Sciences Research Institute Berkeley California; email: iosevich @msri.org 